\title{
High frequency of PDGFRA and MUC family gene mutations in diffuse hemispheric glioma, H3 G34-mutant: a glimmer of hope?
}

Wanming $\mathrm{Hu}^{1 \dagger}$, Hao Duan ${ }^{2 \dagger}$, Sheng Zhong ${ }^{2 \dagger}$, Jing Zeng ${ }^{1 *}$ and Yonggao Mou ${ }^{2 *}$

\begin{abstract}
Background: Diffuse hemispheric glioma H3 G34-mutant (G34-DHG) is a new type of pediatric-type diffuse highgrade glioma in the fifth edition of the WHO Classification of Tumors of the Central Nervous System. The current treatment for G34-DHG involves a combination of surgery and conventional radiotherapy or chemotherapy; however, the therapeutic efficacy of this approach is not satisfactory. In recent years, molecular targeted therapy and immunotherapy have achieved significant benefits in a variety of tumors. In-depth understanding of molecular changes and immune infiltration in G34-DHGs will help to establish personalized tumor treatment strategies. Here, we report the clinicopathological, molecular and immune infiltration characteristics of G34-DHG cases from our center along with cases from the HERBY Trial and the Chinese Glioma Genome Atlas database (CGGA).
\end{abstract}

Methods: Hematoxylin-eosin (HE) and immunohistochemistry (IHC) staining were used to present the clinicopathological characteristics of 10 Chinese G34-DHG patients treated at our institution. To address the molecular characteristics of G34-DHG, we performed whole-exome sequencing (WES) and RNA sequencing (RNA-seq) analyses of 5 patients from our center and 3 Chinese patients from the Chinese Glioma Genome Atlas (CGGA) database. Additionally, 7 European G34-DHG patients from the HERBY Trail were also subjected to analyses, with 7 cases of WES data and 2 cases of RNA-seq data. Six G34-DHG patients from another organization were used as external validation.

Results: WES showed a high frequency of PDGFRA mutation in G34-DHGs (12/15). We further identified frequent mutations in MUC family genes in G34-DHGs, including MUC16 (8/15) and MUC17 (8/15). Although no statistical difference was found, PDGFRA mutation tended to be an indicator for worse prognosis whereas MUC16/MUC17 mutation indicated a favorable prognosis in G34-DHGs. RNA sequencing results revealed that most G34-DHG are considered to be immune cold tumors. However, one patient in our cohort with MUC16 mutation showed significant immune infiltration, and the total overall survival of this patient reached 75 months.

Conclusions: Our results demonstrate that G34-DHG is a new high-grade glioma with high frequency of PDGFRA and MUC gene family mutations. PDGFRA may serve as an indicator of poor prognosis and an effective therapeutic target. Moreover, MUC16 tends to be a favorable prognostic factor and indicates high immune infiltration in certain

\footnotetext{
*Correspondence: zengjing@sysucc.org.cn; mouyg@sysucc.org.cn

'Wanming Hu, Hao Duan and Sheng Zhong contributed equally to this work

${ }^{1}$ Department of Pathology, State Key Laboratory of Oncology in South

China, Collaborative Innovation Center for Cancer Medicine, Sun Yat-Sen University Cancer Center, Guangzhou, China

${ }^{2}$ Department of Neurosurgery, State Key Laboratory of Oncology in South China, Collaborative Innovation Center for Cancer Medicine, Sun Yat-Sen University Cancer Center, Guangzhou, China
}

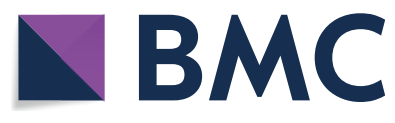

(c) The Author(s) 2022. Open Access This article is licensed under a Creative Commons Attribution 4.0 International License, which permits use, sharing, adaptation, distribution and reproduction in any medium or format, as long as you give appropriate credit to the original author(s) and the source, provide a link to the Creative Commons licence, and indicate if changes were made. The images or other third party material in this article are included in the article's Creative Commons licence, unless indicated otherwise in a credit line to the material. If material is not included in the article's Creative Commons licence and your intended use is not permitted by statutory regulation or exceeds the permitted use, you will need to obtain permission directly from the copyright holder. To view a copy of this licence, visit http://creativecommons.org/licenses/by/4.0/. The Creative Commons Public Domain Dedication waiver (http://creativeco mmons.org/publicdomain/zero/1.0/) applies to the data made available in this article, unless otherwise stated in a credit line to the data. 
patients, and these findings may provide a new direction for targeted therapy and immunotherapy of patients with G34-DHGs.

Keywords: Diffuse hemispheric glioma, H3G34R/N, H3K27M, PDGFRA, MUC, Immune infiltration, Survival

\section{Background}

Pediatric-type diffuse high-grade glioma (pHGG) is a highly aggressive brain tumor with a poor prognosis that accounts for approximately $8-15 \%$ of all central nervous system tumors in children and adolescents [1]. The widespread use of high-throughput sequencing, genetic profiling and epigenetic analysis has greatly increased our understanding of the cell origin, pathogenesis and biological characteristics of pHGG. Two types of heterozygous somatic mutations in $H 3 F 3 A$, which encodes histone H3, were firstly identified in pHGG in 2012 [2]. These mutations lead to an amino acid substitution at key residues (K27M or G34R/G34V). Subsequent studies found that K27M mutation presented in both H3F3A and HIST1H3B/C genes, but G34R/V-mutant gliomas were characterized by recurrent glycine-to-arginine/ valine alterations at codon $34(\mathrm{G} 34 \mathrm{R} / \mathrm{V})$ only in the H3F3A gene. The fifth edition of the WHO Classification of Tumors of Central Nervous System in 2021 defined the standard name of G34R/V-mutant gliomas as diffuse hemispheric glioma H3 G34-mutant, classified as WHO grade 4 .

Most diffuse hemispheric glioma H3 G34-mutant tumors (G34-DHGs) carry TP53 mutations and ATRX deletions [2], with hypermethylation at the OLIG2 locus, leading to the absence of OLIG2 expression [3]. The current treatment for these tumors is surgery, radiotherapy and chemotherapy. However, the efficacy of this therapeutic strategy is not satisfactory. In-depth understanding of the molecular changes and immune infiltration in G34-DHGs will help to establish personalized tumor treatment strategies.

In this study, we conducted a comprehensive analysis of G34-DHGs at the clinicopathological and genetic level. Our research uncovered new molecular characteristics of this unique tumor, which provide potential opportunities to develop individualized therapy.

\section{Methods}

\section{Patient cohort}

The institutional ethical committee of Sun Yat-sen University Cancer Center (SYSUCC) approved this study, and written informed consent was obtained from all patients. A flow diagram of patient selection is shown in Additional file 1: Figure S1.

Ten H3F3A G34-mutated patients were selected from the clinicopathology database on the basis of $H 3 F 3 A$
Sanger sequencing results, including nine patients with G34R mutation and one patient with G34V mutation. Pathological specimens of all patients were independently reviewed by two senior neuropathologists according to the criteria of the 2021 WHO Classification of Tumors of Central Nervous System [4]. All patients met the criteria for diffuse hemispheric glioma, H3 G34mutant. Clinicopathological characteristics of patients including age, sex, chief complaint, site of lesion, treatment, IDH/TERT/MGMT promoter methylation status, and survival status were retrospectively reviewed.

Five cases had sufficient fresh tumor samples for wholeexome sequencing (WES) and RNA sequencing (RNAseq); freshly frozen tumor tissues were subjected to WES and RNA-seq analyses, and corresponding blood samples were subjected to WES analyses. We also obtained WES and RNA-seq data of three Chinese patients from the Chinese Glioma Genome Atlas (CGGA) database and seven European patients from the HERBY Trial (study BO25041; clinicaltrials.gov NCT01390948).

For survival analysis, we examined overall survival (OS) of the total 20 patients with G34-DHG, along with 21 cases with $I D H$-mutant high-grade glioma and 34 cases with H3K27M-mutant diffuse midline glioma at our institution.

\section{Immunohistochemistry}

Immunohistochemical staining was performed using an immunostaining system (BenchMark ULTRA system, Ventana-Roche, Switzerland) with primary antibodies against GFAP (1:200, ZSGB-BIO, Beijing, China), OLIG2 (1:100, ZSGB-BIO), ATRX (1:200, ZSGB-BIO), IDH1 R132H (1:100, MX031, Fuzhou, China), H3K27M (1:1000, Millipore, Temecula, CA, USA), p53 (1:1000, Dako, Glostrup, Denmark) and Ki67 (1:200; Dako). Appropriate positive controls were included.

\section{Methylation-specific PCR analysis}

Methylation-specific PCR was conducted using an EZ DNA Methylation Kit (Zymo Research, Irvine, CA, USA) to determine the methylation status of the MGMT promoter, as described previously [5].

\section{WES}

DNA was extracted from fresh tissues, paraffin-embedded tissue and peripheral blood. WES was performed using a targeted capture approach with the Agilent 
SureSelect Human All Exon Kit (Santa Clara, CA, USA) followed by massively parallel sequencing of enriched fragments on the Illumina HiSeq2500 by Gene Denovo Biotechnology Co. Tumor and corresponding blood leukocyte DNA samples had an average sequencing depth of the target exonic region of $>200 \times$. For a big panel targeting 539 and a small panel targeting 135 tumor genes, the sequencing depth was $2000 \times$.

\section{Somatic variant identification}

All the sequence reads were mapped according to the human reference genome GRCh37 using BWA-MEM with default parameters. Following the GATK standard protocol, realignment and recalibration were performed for BAM files after removing PCR duplicates. Singlenucleotide variations (SNVs) were identified with MuTect in the GATK suite. Using white blood cell data as controls, tumor sample data were then input into GATK to call somatic mutations (SNV). Finally, maftools was employed to visualize the mutational landscape of the results.

\section{Immune infiltration score calculation}

To de-convolute immune components of tumor samples, the TIMER online tool (http://cistrome.org/TIMER/) was employed to evaluate the immune cell infiltration score using default settings. The immune infiltration score was later visualized and plotted by the $\mathrm{R}$ studio function pheatmap.

\section{Statistical analysis}

Statistical analysis was performed using IBM-SPSS Statistics version 18.0 (IBM, NY, USA). Kaplan-Meier survival curves were generated to estimate OS. Survival differences were analyzed by the log-rank test.

\section{Results}

\section{Clinical characteristics of G34-DHGs}

The clinical characteristics of the 10 patients with G34DHGs from SYSUCC are summarized in Table 1. The patient group included four male patients and six female patients. The patient age at initial diagnosis ranged from 13 to 25 years, and the median patient age was 20.5 years. The symptoms were the classic clinical features of brain tumors, including dizziness, headache, nausea, vomiting, weakness of right limbs and generalized tonicclonic seizures dependent on the site of the lesion. All lesions were primarily located in cerebral hemispheres; the lesions were in the right hemisphere in five patients, the left hemisphere in four patients and bilateral hemisphere in one patient. Tumor invasion in the frontal lobe was detected in eight patients, and invasion in the parietal lobe was detected in three patients; temporal lobe involvement was observed in two patients and insular lobe involvement was observed in two patients. In addition, corona radiata, basal ganglia or corpus callosum were infiltrated by tumors in four patients.

For initial treatment, gross total resection (GTR) was performed in five patients, whereas four patients received subtotal resection and one patient only received biopsy. For postoperative adjuvant treatment, nine patients received adjuvant concurrent chemotherapy and radiation therapy (CCRT), and one patient who was included in a clinical trial received 12 courses of dianhydrodulcitol. Among the nine patients receiving CCRT, five patients further received maintenance temozolomide (TMZ) treatment and one patient received four courses of TMZ plus cisplatin followed by maintenance TMZ treatment.

Seven patients showed recurrent disease after the initial treatment, and four patients died. Four patients who initially received subtotal resection chose conservative treatment after recurrence, and two died at 6 and 16 months after the initial operation. The remaining three patients with recurrent disease received a second operation, and two of them further received adjuvant therapy after the second operation. Patient 9 received TG02, a novel pyrimidine-based multi-kinase inhibitor of CDKs together with JAK2 and FLT3, after STR for recurrent disease. This patient died at 1 year after recurrence with an OS of 17 months. Patient 1, who was diagnosed with methylated MGMT promoter after initially receiving GTR followed by CCRT and 12 courses of maintenance TMZ treatment, then again underwent GTR followed by radiotherapy and 12 courses of maintenance TMZ treatment for her recurrent disease; this patient achieved the longest OS of 75 months. Moreover, three patients who were also diagnosed with methylated MGMT promoter after initially receiving GTR followed by CCRT and maintenance TMZ treatment were free of recurrence after at least 18 months since the initial operation. Intriguingly, two of the three patients without recurrent disease continuously received maintenance TMZ treatment (ongoing), including patient 4 , who initially received only biopsy. These results indicated that only GTR and longterm maintenance TMZ treatment might benefit patients with G34-DHG in conventional treatment.

\section{Pathological characteristics of G34-DHGs}

Histological features including necrosis, nuclear atypia, mitotic activity and vascular characteristics were assessed. Histopathologic examination of the surgical specimens from our cohort showed high-grade glioma morphology (Fig. 1). All cases showed high cell density, featuring nuclear atypia, mitotic activity and cellular pleomorphism with focal gemistocytic cells and multinuclear giant cells. Most cases were glioblastoma 


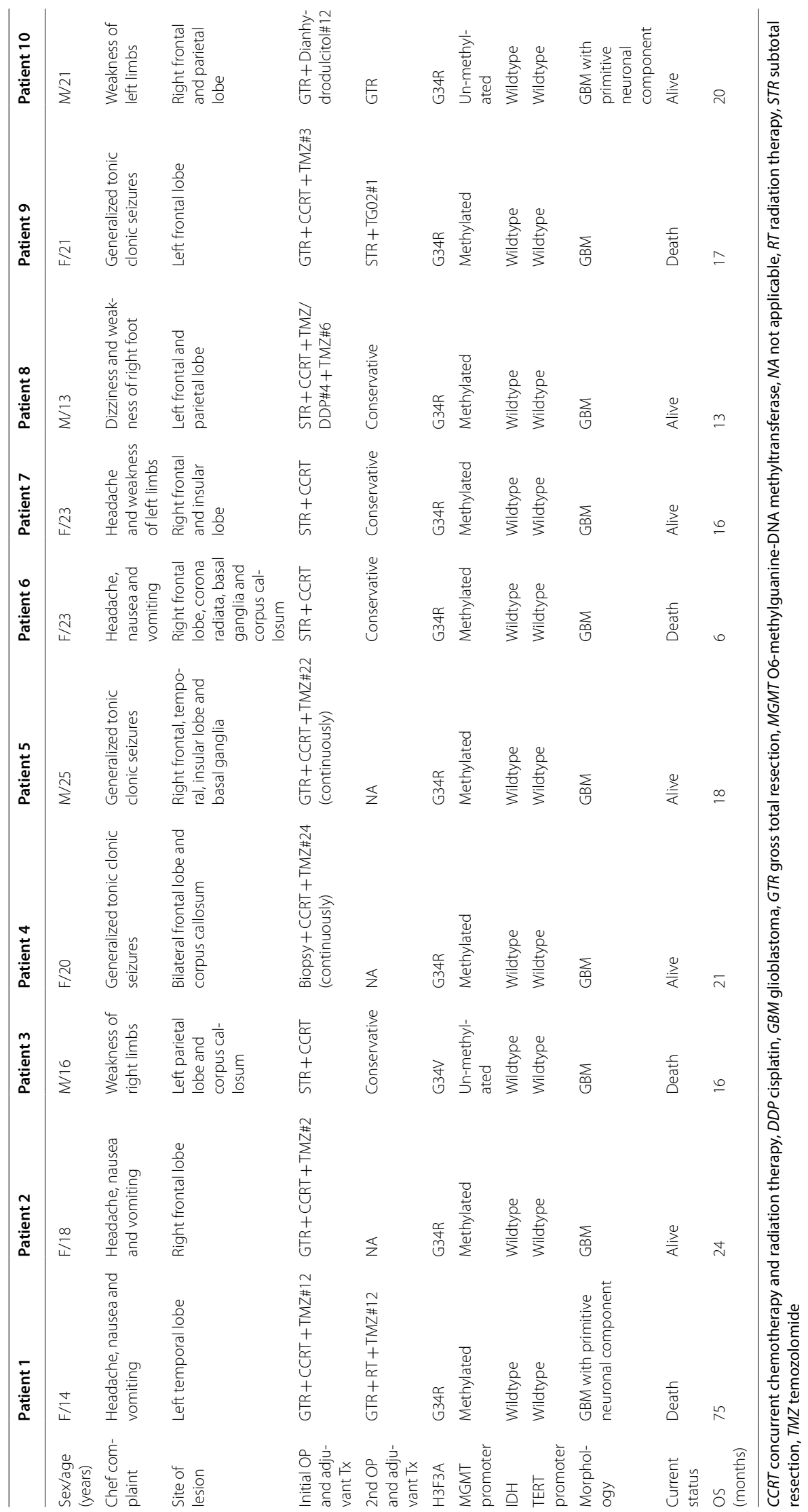




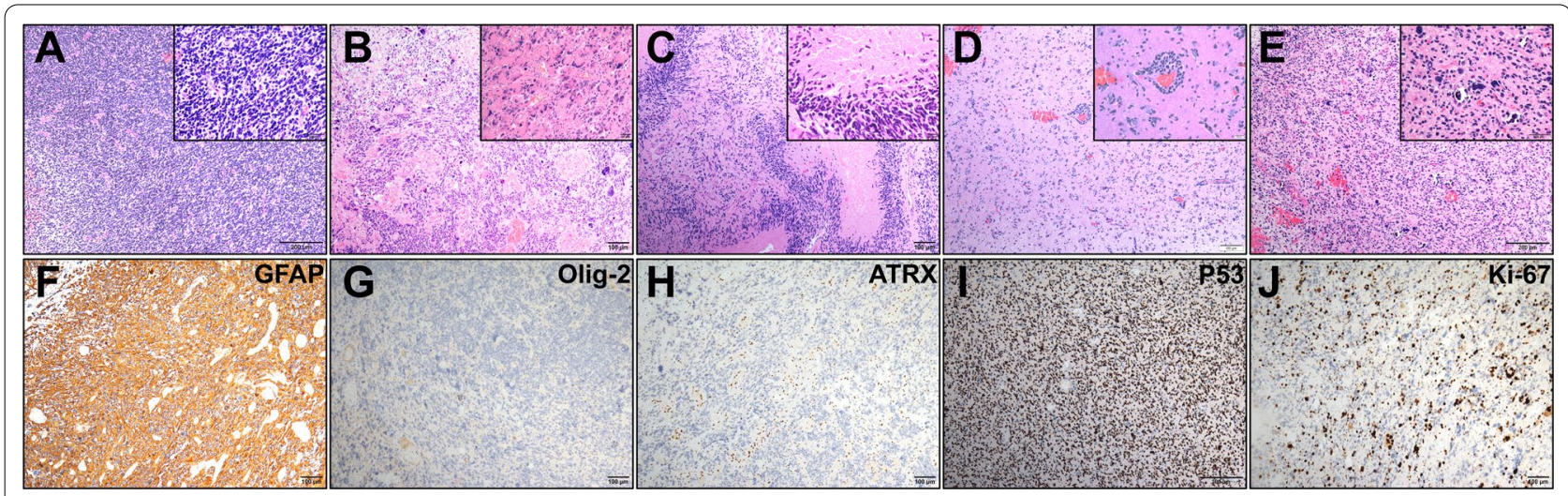

Fig. 1 Histological analysis of diffuse hemispheric glioma H3 G34-mutant specimens. Hematoxylin and eosin staining shows embryonal appearance/small cell glioblastoma-like morphology (A), giant cell glioblastoma-like morphology (B), palisade necrosis (C), perivascular growth pattern (D) and calcification (E). F-J Immunohistochemistry for the indicated proteins

(GBM)-like with microvascular proliferating and/or palisade necrosis and two cases showed focal embryonal appearance. However, calcification, perivascular growth pattern and perineuronal satellitosis, which rarely appears in GBM, were also observed (Fig. 1E, F). All cases showed GFAP expression and negative expression of OLIG2; most cases were negative for ATRX (9/10) and most showed diffuse strong p53 positivity (8/10). The Ki67 labeling index was high, ranging from 20 to $60 \%$. Sanger sequencing revealed that all cases were $I D H$ wild-type and TERT promoter wild-type (Table 1). Methylation PCR revealed that most cases showed MGMT promoter methylation $(8 / 10)$ (Table 1$)$.

\section{Mutational landscape of G34-DHGs}

To investigate the mutational landscape of G34-DHGs, we performed WES on five fresh samples and matched peripheral blood leucocytes from our cohort. The average depths of targeted exome regions in tumors and matched blood samples were $400 \times$ and $100 \times$, respectively. More than $98.69 \%$ of the targeted regions were covered sufficiently for confident variant calling $(\geq 10 \times$ depth). We included three G34-DHG cases from the CGGA and seven G34-DHG cases from the HERBY trial (study BO25041; clinicaltrials.gov NCT01390948). The total somatic mutations of the 15 G34-DHG samples are listed in Fig. 2.

The mutational frequencies, mutation types and clinical features of the 15 G34-DHGs are displayed in Fig. 2A. TP53 (13/15 87.0\%), PDGFRA (12/15, 80.0\%) and ATRX $(10 / 15,67.0 \%)$ were the most three frequently mutated genes. Other frequently mutated genes including $M U C 17$ (8/15, 53.0\%), MUC16 (8/15, 53.0\%), MUC5B (7/15, 47.0\%), MUС3A (7/15, 47.0\%), OBSCN (6/15, 40.0\%),
$\operatorname{SSPO}(6 / 15,40.0 \%)$ and $D O C K 3(6 / 15,40.0 \%)$ were identified by WES.

Notably, MUC family gene mutations have not been reported in G34-DHGs. The human MUC16 gene is located on chromosome 19p13.2 and the MUC17 gene is located on chromosome $7 \mathrm{q} 22.1$. Out of the eight cases with MUC16 mutations, $87.5 \%$ (7/8) of the cases had missense mutations, and the remaining case had a frame_shift_ins mutation. Moreover, all eight cases with MUC17 mutations had missense mutations.

\section{Somatic SNVs and indels}

A total of 8285 exonic mutations were identified in the 15 G34-DHG patients. Of these mutations, 7187 were missense mutations, 448 were nonsense mutations, 153 were frameshift deletions, 313 were frameshift insertions, 10 were in_frame_del mutations, 1 was an in frame_ins mutation and 173 were splice site mutations. We removed 1561 silent variants with unknown function. The predominant types of nucleotide substitutions in SNVs in G34-DHGs were $\mathrm{C}>\mathrm{T} / \mathrm{G}>\mathrm{A}$ transitions and $\mathrm{C}>\mathrm{A} / \mathrm{G}>\mathrm{T}$ transversions.

\section{Oncogenic pathways analysis}

We identified multiple pathways of somatic mutated genes in G34-DHGs using the oncogenic pathways module of the $\mathrm{R}$ package maftools. Crucial signal transduction pathways included the RTK-RAS, NOTCH, WNT, Hippo, PI3K, TP53, MYC and Cell_Cycle pathways (Fig. 2B). Exome sequencing revealed that 14 of 15 cases had changes in the receptor tyrosine kinase RTK-RAS pathway, involving $42 / 84$ pathway genes (including $P D G$ FRA, MET, BRAF, ERF, FGFR1 and NF1). KEGG pathway analysis revealed that many of the mutated genes were involved in cancer signal transduction. Mutant 


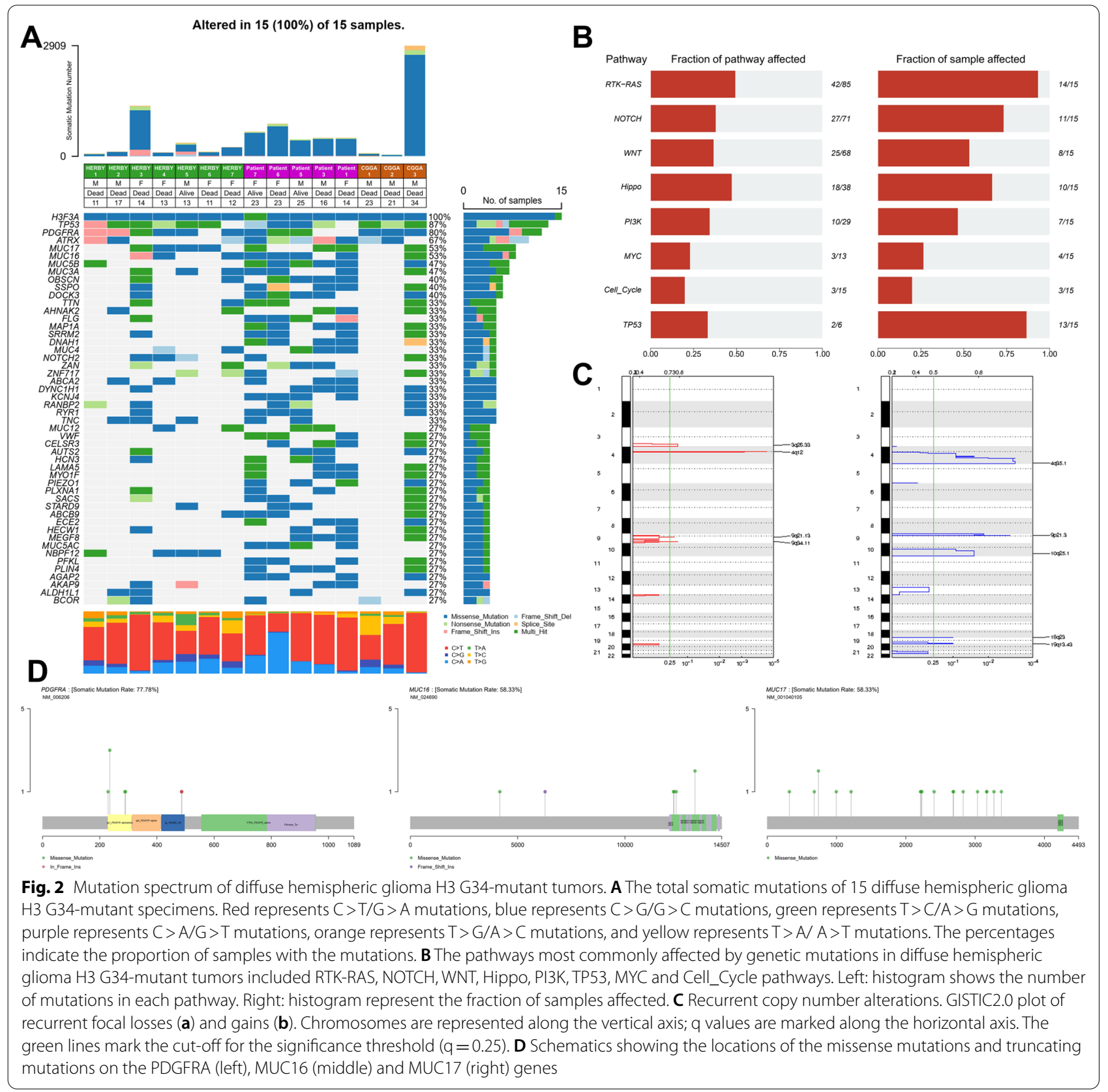

genes may promote tumor cell proliferation and escape apoptosis through cascade reaction.

\section{Copy number alterations (CNAs)}

We conducted somatic CNA analyses in the five SYSUCC cases and seven HEBRY cases. The results identified recurrent gains in chromosomes 3p26.33 (11/12), 4p12 (11/12), 9q21.13 (7/12) and 9q34.11 (4/12). Recurrent losses were identified in chromosomal regions 4 p35.1 (10/12), 10q25.1 (10/12), 19p13.43 (9/12), 18q23 (8/12) and 9 p21.3 (7/12). Loss of somatic CNAs on chromosome 10q25 affects the largest number of genes (1330 genes), including the MGMT locus. Gains of somatic CNAs on chromosome 3p26 affects 396 genes, including $A B C C 5$. Studies have shown that ABCC5 is associated with chemoresistance of astrocytic tumors [6].

\section{PDGFRA mutation and G34-DHGs}

PDGFRA is an important receptor tyrosine kinase in glial development and a recurrent driver in high-grade gliomas [7-9] PDGFRA mutation and the PDGFRA signaling pathway were reported to play potent oncogenic roles 
in G34-DHGs [10]. Our sequencing results also showed that most G34-DHGs had PDGFRA mutation (12/15).

To explore the potential pathways and genes related to PDGFRA mutation in G34-DHGs, we analyzed differentially expressed genes (DEGs) using RNA-Seq data from two G34-DHG patients with wild-type PDGFRA and eight G34-DHG patients with mutated PDGFRA. The results identified 150 DEGs $(|\mathrm{FC}| \geq 2.0$ and $\mathrm{P}<0.05$ ), including 95 down-regulated genes and 55 up-regulated genes. The DEGs between the two groups are shown in a heatmap in Fig. 3A. We identified the top 10 hub genes ranked by degree, including FOS, CXCL8, CXCR1, IL1B, COL1A1, MMP9, FCGR3B, TNF, CCL4 and CCL3. We used MCODE in Cytoscape to identify gene modules in the PPI network and mapped the interaction network of 52 core genes in module 1 (Fig. 3B). The results indicated that the genes were mainly involved with blood microparticles, the phospholipase $\mathrm{C}$-activating $\mathrm{G}$ protein-coupled receptor signaling pathway, substratespecific channel activity, complement and coagulation cascades, the neuroactive ligand-receptor interaction and aldosterone-regulated sodium reabsorption.

We used the DAVID database to analyze the GO and KEGG pathways of the DEGs. KEGG pathway analysis revealed that DEGs were significantly enriched in the extracellular matrix-receptor interaction, cytokinecytokine receptor interaction, PIK3-AKT signaling pathway and chemokine signaling pathway (Fig. 3C). The enriched GO-Biological Process terms included immune and inflammatory response (Fig. 3D).

\section{MUC16 mutation and immune infiltration characteristics of G34-DHGs}

Previous studies indicated that pHGGs with a high mutation load have an elevated neoantigen load and immune response [11, 12]. However, tumors with
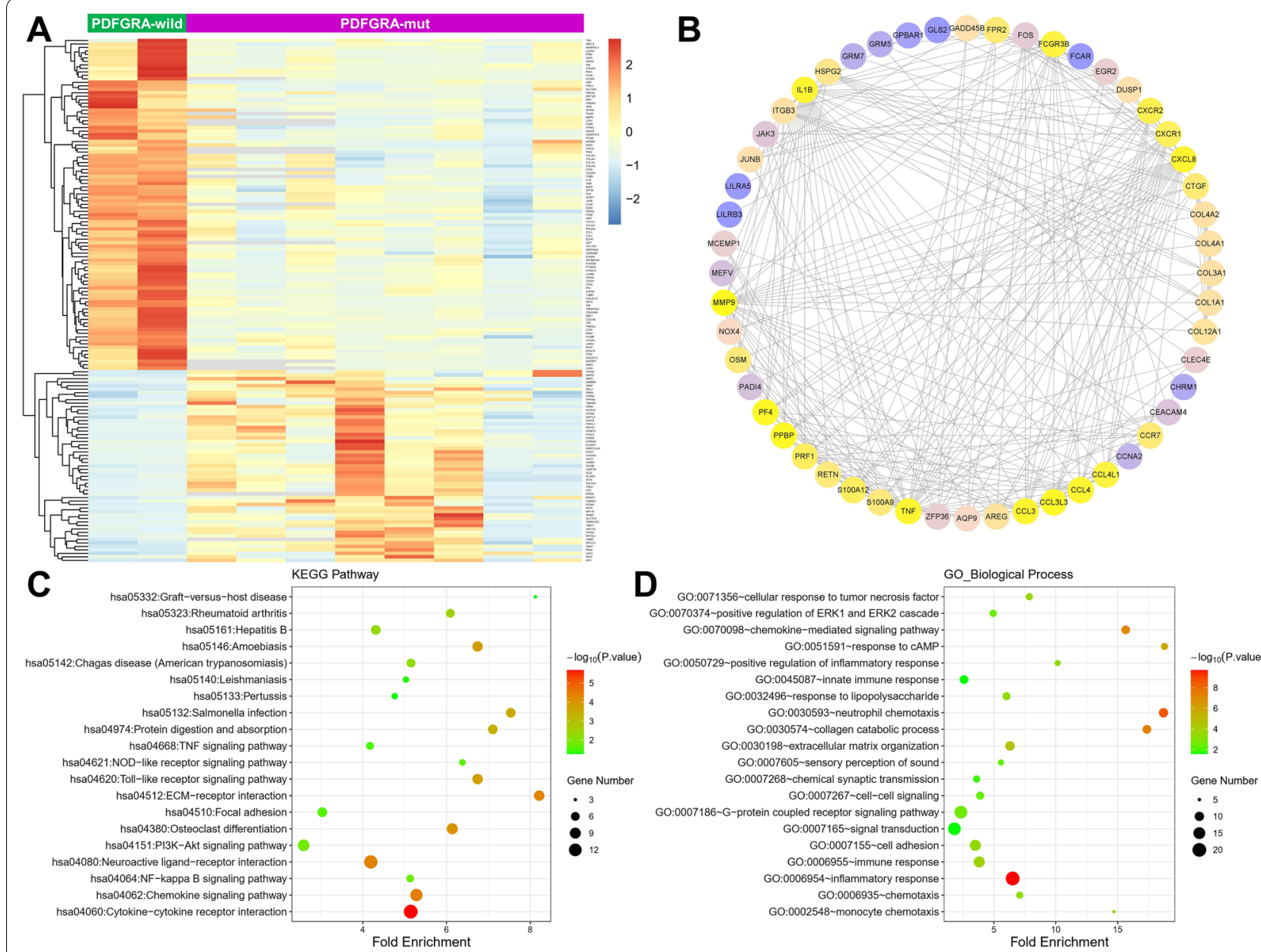

Fig. 3 Analysis of differentially expressed genes in diffuse hemispheric glioma H3 G34-mutant tumors with PDGFRA mutation. Differential gene expression in two G34-DHG patients with wild-type PDGFRA and eight G34-DHG patients with mutated PDGFRA (A). Protein-protein interactions of the differentially expressed genes (B), KEGG pathway analysis (C) and GO-Biological process analysis (D) 
histone $\mathrm{H} 3$ gene mutations are considered as immune cold tumors, which are defined as a lack of CD8 immunoreactivity and lack of tumor-infiltrating lymphocytes [13]. Using the RNA sequencing results, we next analyzed the expressions of immune-related genes in 10 patients with G34-DHGs, including 5 from SYSUCC, 3 from CGGA and 2 of the HERBY cases. The 67 differentially expressed immune-related genes were classified according to CD8 $+\mathrm{T}$ cell, $\mathrm{T}$ cell (general), B cells, monocyte, tumor-associated macrophage, M1 macrophage, M2 macrophage, neutrophil, natural killer cell, dendritic cell, Th1, Th2, Tfh, Th17, Treg and T cell exhaustion markers (Fig. 4). In general, consistent with HERBY Phase II Randomized Trial, we also found G34DHGs were immune cold tumors, with a few exceptions. For instance, the tumor specimen of patient 1 in

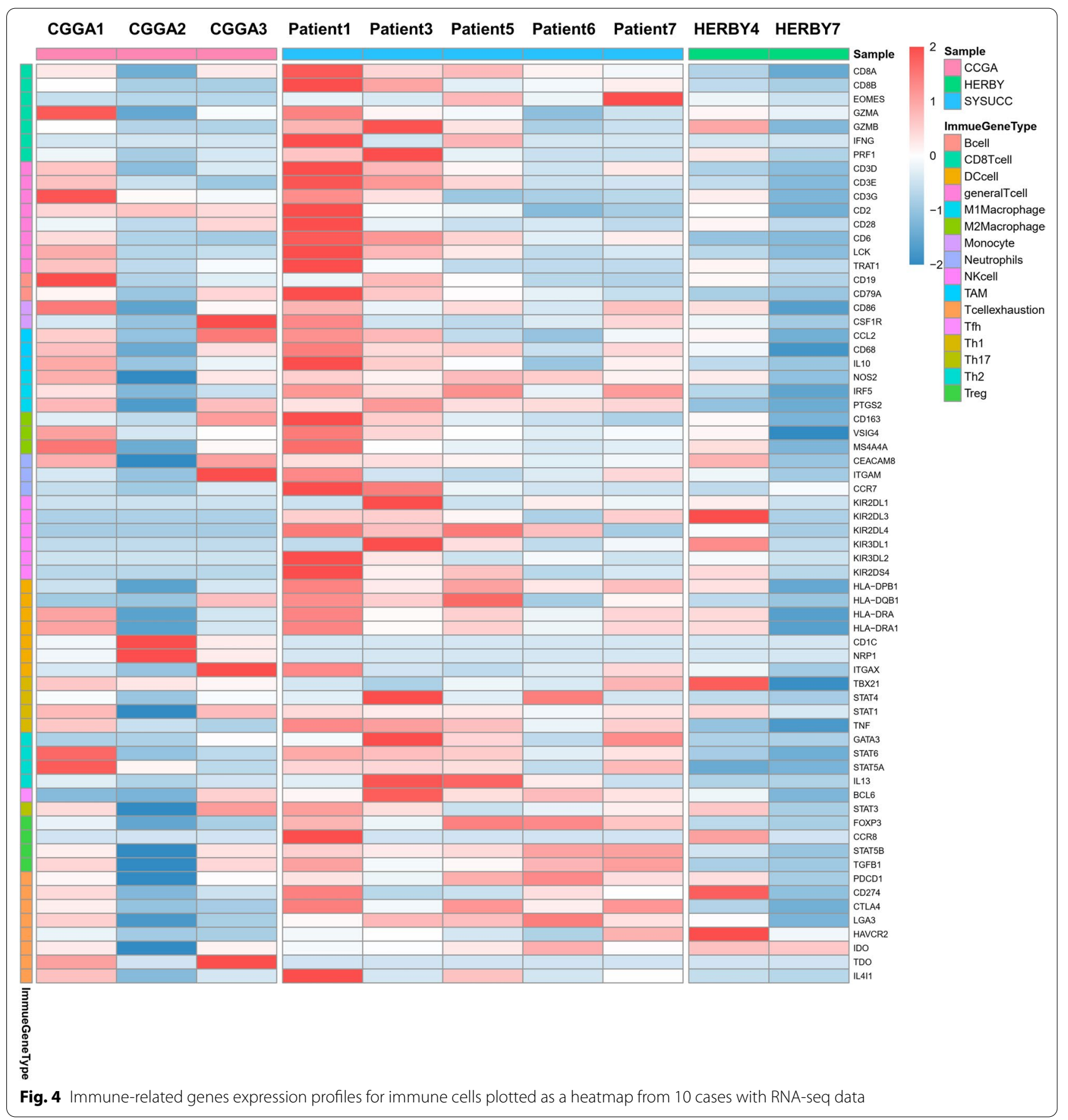


our cohort showed significant immune infiltration with substantial amounts of CD4 and CD8 T cells (Additional file 2: Figure S2). The OS of this patient reached 75 months, which was markedly longer than that of the nine patients with low immune infiltration (mean survival time: 12 months).

A previously published pan-cancer analysis of 30 solid tumor types showed that patients with MUC16 mutations showed higher tumor mutation burden and neoantigen burden, indicating increased tumor immunogenicity, which can predict immune checkpoint inhibitor treatment response. The study included 397 cases of glioblastoma and 61 of these cases (15.37\%) showed MUC16 mutations [14]. Therefore, we wondered whether the MUC16 mutation in G34-DHGs might also be associated with immune infiltration.

We used TIMER 2.0 to analyze the immune cell infiltration of G34-DHGs with MUC16 mutation and G34-DHGs with wild-type MUC16. However, no connection between MUC16 mutation and immune cell infiltration was found; this may be because of the small number of cases and relatively low immune infiltration of G34 glioma. We further analyzed the DEGs between G34-DHGs with MUC16 mutation and G34-DHGs with wild-type MUC16. G Protein-Coupled Receptors (GPCR) signaling relevant proteins MTNR1B, OXTR and PDYN were all low expressed in G34-DHGs with MUC16 mutation (Fig. 5).

\section{Survival analysis of patients with G34-DHGs}

We performed survival analyses of the patients from our center (SYSUCC) (Fig. 6). We found that the OS of patients with H3G34-mutant DHGs was worse than that of patients with IDH-mutant high-grade gliomas, but better than that of patients with H3K27M-mutant DMGs. The mean survival times of patients with IDHmutant high-grade gliomas, G34-DHGs and H3K27M DMGs were 58.4, 53.8 and 18.4 months, respectively $(\mathrm{P}<0.001)$.

We further performed analysis in the overall patient group (patients with G34-DHGs from SYSUCC, CGGA and HERBY Trial). Age ( $\geq 18$ years old vs. $<18$ years old) and sex (male vs. female) did not influence patient prognosis. Although no statistical difference was achieved, Kaplan-Meier curve analyses showed some trends according to race, PDGFRA mutation, MUC16 mutation and MUC17 mutation. The median OS for Chinese patients was 18 months compared with 12 months for Caucasian patients $(\mathrm{P}=0.105)$. Patients with $P D G$ FRA mutation tended to show a shorter OS (median OS: 11.5 months vs. 16 months), and MUC16 and MUC17 mutations both seemed favorable for prognosis (median OS: 15 months vs. 12 months and 16 months vs. 11.5 months, respectively). Importantly, although immune infiltration varied in G34 glioma patients, patient 1 in our cohort who harbored MUC16 mutation
A

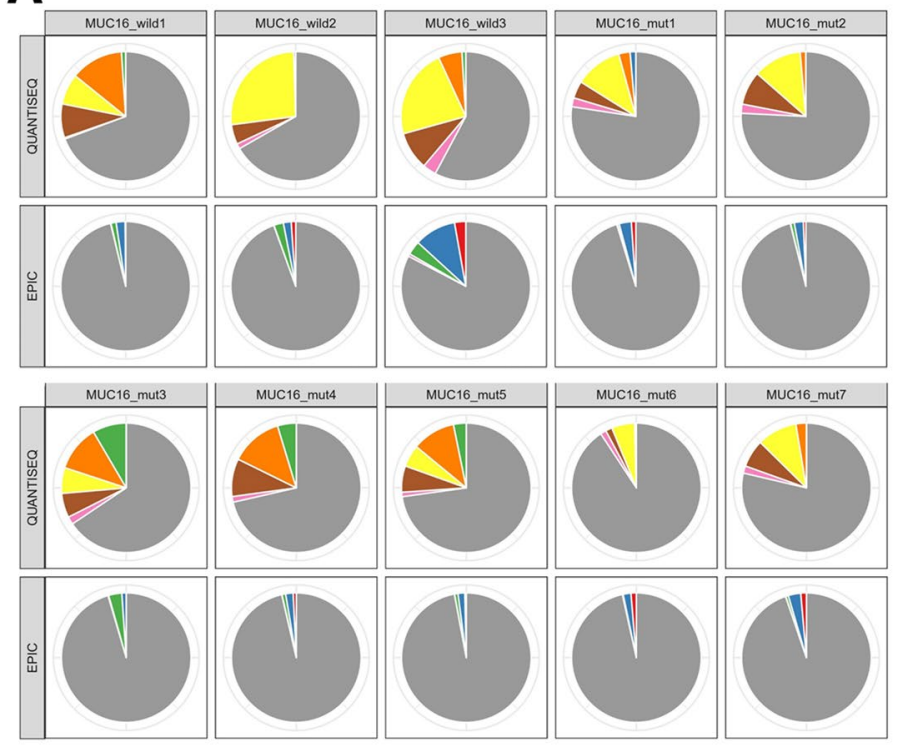

B

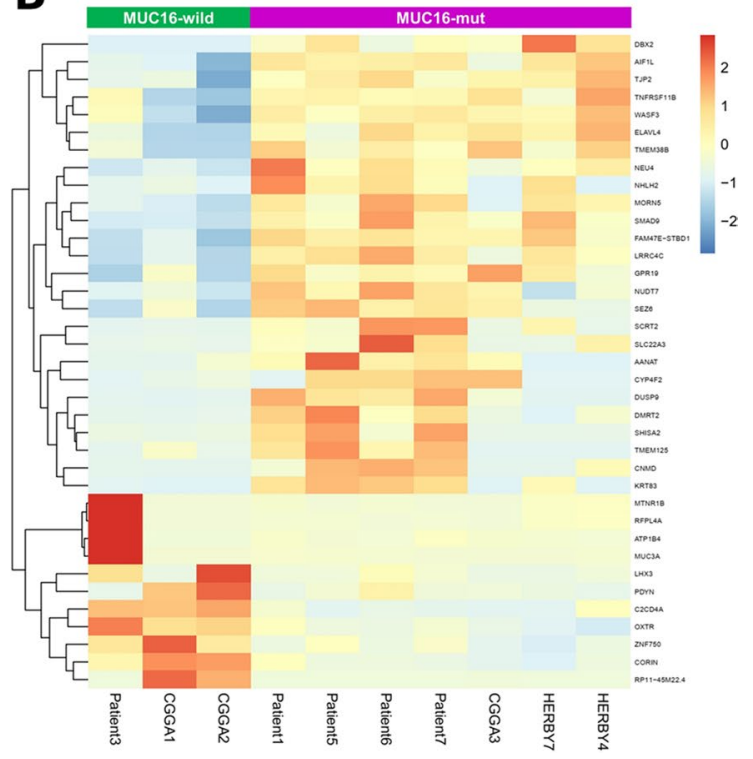

Fig. 5 Immune gene expression signatures and differential genes for MUC16 mutant and wild-type diffuse hemispheric glioma H3 G34-mutant tumors. A A multi-panel pie plot showing the proportion of immune cell types in MUC16 mutant and wild-type samples. The eight immune cell types are highlighted in different colors. B Differential gene expression types in MUC16 mutant and wild-type cases 


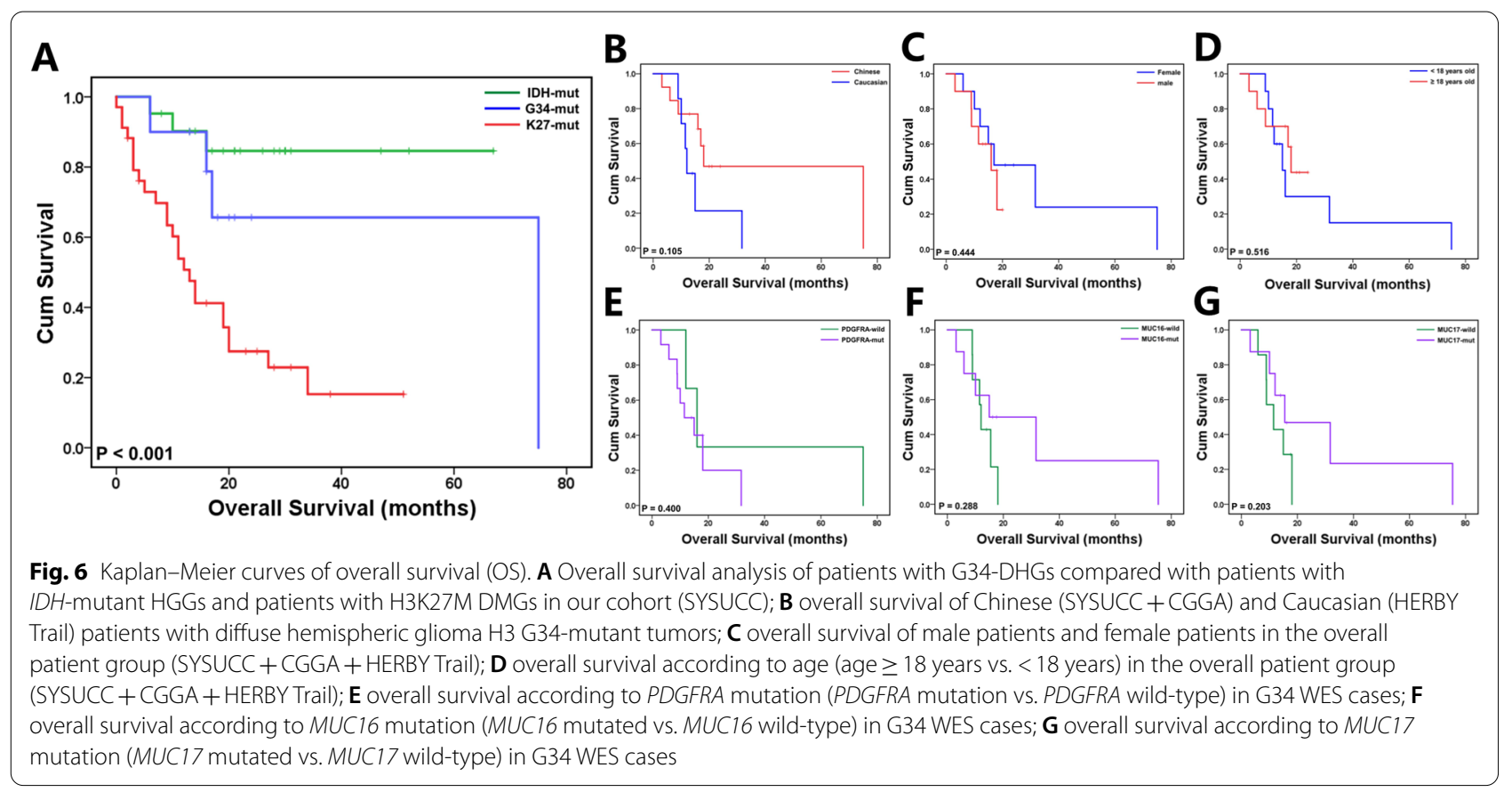

had obviously high immune infiltration and achieved the longest OS of 75 months.

\section{External validation}

To validate that PDGFRA and MUC family gene mutations status in G34-DHGs, we reviewed the reports of tumor-sequencing test from 6 external patients, among which WES, a big panel targeting 539 or a small panel targeting 135 tumor genes were respectively performed in every 2 patients (Table 2). Somatic alterations in PDGFRA were detected in all 6 external patients, including 2 patients having both missense mutations and gene amplification, 1 patient having a missense mutation only, 1 patient having both a missense mutation and a deletioninsertion variant, and 2 patients having in-frame insertions or deletions. For the 2 patients who received WES, missense mutations in MUC4 and MUC12 were detected individually. Unfortunately, the targeted sequencing panels did not include MUC family genes in the panel.

\section{Discussion}

Diffuse hemispheric glioma H3 G34-mutant is a newly recognized tumor type in the 2021 WHO Classification of Tumors of the Central Nervous System. This tumor is a malignant infiltrative glioma that typically occurs in the cerebral hemispheres and harbors a missense mutation in

Table 2 Somatic alterations in PDGFRA and MUC family in G34-DHGs of 6 external patients

\begin{tabular}{|c|c|c|c|c|c|}
\hline Patients & Sex/age (years) & Sequencing test & Somatic alterations in PDGFRA & $\begin{array}{l}\text { Somatic } \\
\text { alterations in MUC } \\
\text { family }\end{array}$ & Other important molecular changes \\
\hline ExP1 & $M / 22$ & WES & p.E387del & MUC4 p.V1961L & ATRX p.K1584lfs, EGFR amplification \\
\hline ExP2 & $F / 18$ & WES & $\begin{array}{l}\text { p.L275S } \\
\text { Amplification }\end{array}$ & MUC12 p.T1733A & ATRX p.Q2168Rfs, TP53 p.R306\&p.R342 \\
\hline ExP3 & $F / 37$ & 539 genes panel & $\begin{array}{l}\text { p.E279_A280delinsT } \\
\text { p.Y288C }\end{array}$ & N/A & $\begin{array}{l}\text { TP53 p.W53\&p.R342 } \\
\text { CDKN2A deletion }\end{array}$ \\
\hline ExP4 & $\mathrm{M} / 10$ & 539 genes panel & $\begin{array}{l}\text { p.C290G } \\
\text { Amplification }\end{array}$ & N/A & $\begin{array}{l}\text { ATRX c.5955_5956+2del } \\
\text { TP53 p.E171del }\end{array}$ \\
\hline ExP5 & $M / 21$ & 135 genes panel & p.Q246_Y249delinsH & N/A & $\begin{array}{l}\text { ATRX p.R781 } \\
\text { TP53 p.V147Lfs }\end{array}$ \\
\hline ExP6 & $M / 32$ & 135 genes panel & p.G286E & N/A & $\begin{array}{l}\text { ATRX c.5567 } \\
\text { TP53 c.524 }\end{array}$ \\
\hline
\end{tabular}


the $H 3 F 3 A$ gene that results in a G34R/V substitution in histone H3. The histological heterogeneity of G34-DHGs has been previously reported [15]. In general, most cases show high-grade anaplastic features, with microscopic features of anaplastic astrocytoma, GBM or embryonic tumors (primitive neuroectodermal tumor-like features) or even anaplastic pleomorphic xanthoastrocytoma [16]. Therefore, misdiagnosis of cases is likely if clinicians and pathologists rely solely on histological diagnosis. Consistent with these findings, most cases in our center also presented as GBM-like and with/without a focal embryonal appearance. However, calcification, perivascular growth pattern and perineuronal satellitosis, which rarely appear in GBM or called 'secondary structures', were observed $[3,17]$. We further analyzed the immunohistochemical and characteristics of H3G34-mutant diffuse gliomas. Lack of OLIG2 expression is one of the characteristics of this tumor and was previously reported in 100\% of G34 DHGs [18]. We also observed loss of OLIG2 expression in all of our cases. In addition, loss of ATRX expression and $\mathrm{p} 53$ overexpression are also frequently observed in this tumor type. The H3F3A G34R/V mutation is associated with a high frequency of MGMT methylation, but this is mutually exclusive with IDH or TERT promoter mutation.

Significantly, we found frequent PDGFRA mutation $(12 / 15)$ in the G34-DHGs in our study. PDGFRA is a classical receptor tyrosine kinase with five extracellular immunoglobulin-like structures and responsive to platelet-derived growth factor (PDGF) [19], and this protein plays an important role in cell determination, cell proliferation and migration during neural development and adult neurogenesis $[19,20]$. In glioma, somatic alterations of PDGFRA include missense mutations, inframe insertions or deletions, gene amplification and fusion. A high proportion of gliomas, especially TERTp wild-type GBM, are associated with somatic alterations in PDGFRA. Recurrent PDGFRA mutations have also been found in low-grade neurotumors associated with refractory seizures, called septal dysplasia neuroepithelial tumors or mucinous glial neuronal tumors [21]. PDGFRA gene amplification is present in approximately one-third of HGGs, and most tumors with PDG$F R A$ gene amplification have genomic deletions of exons 8 and 9[22]. Ozawa et al. reported an adult case with a gene fusion between PDGFRA and VEGFR2 [23]. Point mutations are located in the extracellular domain, transmembrane domain and kinase domain of the PDGFRA receptor. These oncogenic mutations lead to constitutively activated PDGFR $\alpha$, thereby activating downstream signal transduction [24]. Aberrant PDGFRA signaling in gliomas leads to activation of the Ras-Raf-MEK-ERK pathway [25]. Gain-of-function PDGFRA variants trigger multiple tumor-promoting signaling cascades, including phospholipase $\mathrm{C} \gamma$ (PLCY), phosphatidylinositol-3-kinase (PI3K), mitogen-activated protein (MAP) kinase, and signal transduction and Activators of Transcription (STATs) [26, 27]. Targeting PDGFRA by tyrosine kinase inhibitors (TKIs) or antibodies showed promising antitumor effects in patients with various PDGFR-driven extracranial tumors and pre-clinical models of gliomas [28-31]. In a glioma subset with histone H3 mutation (H3K27M DMGs), PDGFRA amplification and mutation were reported together with histone $\mathrm{H} 3.3$ mutation. Li et al. found that 18 cases of 112 midline glioma patients had PDGFRA mutations [32]. Both our study and Chen's study found high frequencies of PDGFRA mutations in G34-DHGs [10]. PDGFRA mutation was present in 12 out of 15 cases $(80 \%)$ in our study group, and Chen et al. extensively analyzed 95 cases of HGGs with H3.3G34R/V mutation and found that PDGFRA mutations presented in $44 \%$ of all tumors and $81 \%$ of recurrent tumors, they speculated that PDGFRA mutant G34 tumors have expanded astrocytic compartments that are conducive to maintaining the active chromatin conformation state of the GSX2 enhancer to maintain high PDGFRA expression and continue to promote the carcinogenic state [10]. In GBM and in $I D H$ mutant lower-grade (WHO Grades II/III) glioma, PDGFRA amplification is associated with shorter progression-free survival and $O S$ and it is an independent prognostic factor [33]. PDGFRA amplification was also an indicator of poor prognosis in H3K27M DMGs [34, 35]. However, the prognostic implication of PDGFRA mutation has not been extensively studied. Our data suggested that PDGFRA mutation may be an indicator for poor prognosis in G34-DHGs, but the results were not statistically significant; this may because of the relatively small number of G34 cases in our cohort.

Another important finding included the MUC family genes were also frequently mutated in G34-DHGs. The MUC gene family encodes mucins, a type of high molecular weight glycoprotein [36]. There are $21 \mathrm{MUC}$ genes in the human genome that encode secreted and membrane-type mucins [37]. Increasing evidence has shown that MUC proteins play an important role in regulating tumor cell proliferation, growth, apoptosis and chemical tolerance [38, 39]. MUC gene mutation analysis showed that MUC16 (OMIM 606154) has the highest mutation frequency in G34-DHGs, followed by $M U C 17$. A pan-cancer analysis involving somatic mutations of 10,195 samples and mRNA expression profiles of 9850 samples for 30 solid tumors found a greater abundance of immune cells in the microenvironment of MUC16-mutated tumors, and MUC16 mutation was associated with factors associated with response to immune checkpoint inhibitor therapy[14]. Tumors with 
MUC16 mutations exhibited a higher tumor mutational burden and more abundant neoantigen compared with that of MUC16 wild-type tumors, indicating increased tumor immunogenicity. MUC16-mutated tumors were characterized by upregulated expression of T-effector and interferon- $\gamma$ gene signature, a hallmark of preexisting immunity associated with pronounced benefit from checkpoint blockade. An additional hallmark of MUC16mutated tumors is the augmented expression of multiple inhibitory checkpoints including LAG3 and others, suggesting potential adaptive immune resistance to antiPD-1/PD-L1 therapies and that additional inhibitory pathways beyond the PD-1/PD-L1 axis might be targets. Although we discovered a high frequency of MUC16 mutation in G34-DHGs, only one case in the MUC16 mutation group showed abnormal high immune infiltration with abundant tumor-infiltrating lymphocytes and this patient survived up to 75 months. We did not find a relationship between $M U C 16$ and immune infiltration in G34-DHGs, which may be from the small sample numbers and immune-cold characteristics of G34-DHGs.

Regarding survival analysis, one study reported that the survival of patients with G34-DHGs was as poor as survival of patients with K27M DMGs [13]. However, all the cases were children in the study and the sample size was small, which may have resulted in bias. Our conclusion is consistent with most studies, showing that the OS of patients with H3 G34-mutant DHGs was worse than the survival of patients with IDH-mutant GBMs, but better than the survival of patients with H3 K27M-mutant DMGs; this may be partly because of the high frequency of MGMT promoter methylation in these tumors and favorable response to TMZ chemotherapy [8].

It is clear that histone mutations represent clearly defined entities within an umbrella HGG classification, and will require therapeutic development and clinical trials distinct. Compared with H3K27M DMGs, G34-DHGs were not sensitive to ONC-201, an effective therapeutic drug for H3K27M-DMGs [40]. Most G34-DHG patients had MGMT promoter methylation, and the rate was higher than the low incidence rate in H3K27M-DMGs (only one case had MGMT promoter methylation in our cohort, 1/34; data not shown), indicating that GTR and long-term maintenance TMZ treatment might benefit patients with G34-DHGs. Most cases also had PDGFRA mutations, and therefore the PDGFRA signaling pathway could be a potential therapeutic target. In addition, immunotherapy may be an option for cases with substantial immune infiltration and MUC16 mutation. These important findings will lay the foundation for the future personalized treatment of G34-DHGs, thereby helping to improve the current scenario in which existing treatment methods have not significantly improved the median survival of these patients.

Our study has some limitations. First, the overall sample size was small, mainly because G34 DHG is a brand-new type of pediatric-type diffuse high-grade gliomas in the latest 2021 WHO classification of tumors of the central nervous system, thus, total number of confirmed cases was small and some G34 DHG cases could be going undiagnosed which lack molecule detection. In addition, the study is mainly descriptive and the significance is possible only for survival analysis of different types of glioma but not yet for the definition of prognostic genetic markers, like PDGFRA and MUC16 and 17 mutations, studies on large sample are needed to confirm the prognostic results of this study. Last, prospective studies such as a follow-up clinical trial is needed to access the effects of the various PDGFRA targeted drugs combined with or without TMZ in G34 DHGs, like phase I study using crenolanib to target PDGFR kinase in children and young adults with newly diagnosed DIPG [41].

\section{Conclusions}

G34-DHG is a new high-grade glioma with high frequency of PDGFRA and $M U C$ gene family mutations. PDGFRA may serve as a poor prognostic indicator and an effective therapeutic target for G34-DHGs. Moreover, MUC16 mutation tends to be a favorable prognostic factor and indicates high immune infiltration in certain patients, which may provide a new direction for immunotherapy of G34-DHGs.

\section{Abbreviations}

G34-DHG: Diffuse hemispheric glioma H3 G34-mutant; CGGA: Chinese Glioma Genome Atlas database; HE: Hematoxylin-eosin; IHC: Immunohistochemistry; WES: Whole-exome sequencing; pHGG: Pediatric-type diffuse high-grade glioma; SYSUCC: Sun Yat-sen University Cancer Center; OS: Overall survival; GTR: Gross total resection; CCRT: Concurrent chemotherapy and radiation therapy; TMZ: Temozolomide; GBM: Glioblastoma.

\section{Supplementary Information}

The online version contains supplementary material available at https://doi. org/10.1186/s12967-022-03258-1.

Additional file 1: Figure S1. Diagram of the study flow. We initially examined 230 glioma cases ( $0-35$ years old) from SYSUCC. 30 cases were excluded due to no enough tissue. IDH1/2 Sanger sequencing was performed in 200 cases, revealing 21 IDH mutated and 179 IDH wild-type cases. H3F3A Sanger sequencing revealed 34 cases with H3 K27M mutation, 9 cases with H3 G34R mutation and 1 case with H3 G34V mutation. The 10 G34R/V cases were reviewed by hematoxylin and eosin and immunohistochemical staining. Only five cases had sufficient fresh tumor samples for whole-exome and RNA sequencing. This study also included 
three cases from CGGA database and seven cases from the HERBY Trial analyzed and selected as shown.

Additional file 2: Figure S2. Substantial T cell infiltration in patient 1. (A) Hematoxylin and eosin staining and immunohistochemistry for (B) CD4 and (C) CD8

\section{Acknowledgements}

We thank Gabrielle White Wolf, PhD, from Liwen Bianji (Edanz) (www.liwen bianji.cn/f for editing the English text of a draft of this manuscript.

\section{Authors' contributions}

WMH, JZ and YGM conceived and designed the experiments; HD and SZ analyzed the data; WMH, HD, SZ, JZ and YGM contributed to the writing and revise of the manuscript. All authors read and approved the final manuscript.

\section{Funding}

This work was funded by the National Natural Science Foundation of China (81872324), and the Guangdong Basic and Applied Basic Research Foundation (2020A1515110069). The funders had no role in study design, data collection and analysis, decision to publish, or preparation of the manuscript.

\section{Availability of data and materials}

Data in the study will be deposited in the Research Data Deposit (RDD, http:// www.researchdata.org.cn/) of Sun Yat-sen University Cancer Center (SYSUCC), which will be shared by request from any qualified investigator upon approval by the SYSUCC data request committee. All data generated or analysed during this study are included in this published article. And the data is available from the corresponding author on reasonable request.

\section{Declarations}

\section{Ethics approval and consent to participate}

The present study was approved by the Ethical Committee of the Sun Yat-sen University Cancer Center (SYSUCC) and was carried out in accordance with the Declaration of Helsinki.

\section{Consent for publication}

Written informed consent was provided by all the included subjects.

\section{Competing interests}

The authors declare that they have no competing interests.

Received: 19 September 2021 Accepted: 17 January 2022

Published online: 02 February 2022

\section{References}

1. Jones C, Perryman L, Hargrave D. Paediatric and adult malignant glioma: close relatives or distant cousins? Nat Rev Clin Oncol. 2012;9(7):400-13.

2. Schwartzentruber J, Korshunov A, Liu XY, Jones DT, Pfaff E, Jacob K, Sturm $D$, Fontebasso AM, Quang DA, Tonjes $M$, et al. Driver mutations in histone H3.3 and chromatin remodelling genes in paediatric glioblastoma. Nature. 2012;482(7384):226-31.

3. Korshunov A, Capper D, Reuss D, Schrimpf D, Ryzhova M, Hovestadt V, Sturm D, Meyer J, Jones C, Zheludkova O, et al. Histologically distinct neuroepithelial tumors with histone 3 G34 mutation are molecularly similar and comprise a single nosologic entity. Acta Neuropathol. 2016:131(1):137-46.

4. Louis DN, Perry A, Wesseling P, Brat DJ, Cree IA, Figarella-Branger D, Hawkins C, Ng HK, Pfister SM, Reifenberger G, et al. The 2021 WHO classification of tumors of the central nervous system: a summary. Neuro Oncol. 2021;23(8):1231-51

5. Hu WM, Wang F, Xi SY, Zhang X, Lai JP, Wu HY, Liu LL, Sai K, Zeng J. Practice of the new integrated molecular diagnostics in gliomas: experiences and new findings in a single chinese center. J Cancer. 2020;11(6):1371-82.

6. Bronger H, Konig J, Kopplow K, Steiner HH, Ahmadi R, Herold-Mende C, Keppler D, Nies AT. ABCC drug efflux pumps and organic anion uptake transporters in human gliomas and the blood-tumor barrier. Cancer Res. 2005;65(24):11419-28.

7. Mackay A, Burford A, Carvalho D, Izquierdo E, Fazal-Salom J, Taylor KR, Bjerke L, Clarke M, Vinci M, Nandhabalan M, et al. Integrated molecular meta-analysis of 1,000 pediatric high-grade and diffuse intrinsic pontine glioma. Cancer Cell. 2017;32(4):520-537.e5.

8. Sturm D, Witt H, Hovestadt V, Khuong-Quang DA, Jones DT, Konermann C, Pfaff E, Tonjes M, Sill M, Bender S, et al. Hotspot mutations in H3F3A and IDH1 define distinct epigenetic and biological subgroups of glioblastoma. Cancer Cell. 2012;22(4):425-37.

9. Verhaak RG, Hoadley KA, Purdom E, Wang V, Qi Y, Wilkerson MD, Miller CR, Ding L, Golub T, Mesirov JP, et al. Integrated genomic analysis identifies clinically relevant subtypes of glioblastoma characterized by abnormalities in PDGFRA, IDH1, EGFR, and NF1. Cancer Cell. 2010;17(1):98-110.

10. Chen C, Deshmukh S, Jessa S, Hadjadj D, Lisi V, Andrade AF, Faury D, Jawhar W, Dali R, Suzuki H, et al. Histone H33G34-mutant interneuron progenitors Co-opt PDGFRA for gliomagenesis. Cell. 2020;183(6):16171633.e22.

11. Gojo J, Pavelka Z, Zapletalova D, Schmook MT, Mayr L, Madlener S, Kyr M, Vejmelkova K, Smrcka M, Czech T, et al. Personalized treatment of H3K27M-mutant pediatric diffuse gliomas provides improved therapeutic opportunities. Front Oncol. 2019;9:1436.

12. Indraccolo S, Lombardi G, Fassan M, Pasqualini L, Giunco S, Marcato R, Gasparini A, Candiotto C, Nalio S, Fiduccia P, et al. Genetic, epigenetic, and immunologic profiling of MMR-deficient relapsed glioblastoma. Clin Cancer Res. 2019;25(6):1828-37.

13. Mackay A, Burford A, Molinari V, Jones D, Izquierdo E, Brouwer-Visser J, Giangaspero F, Haberler C, Pietsch T, Jacques TS, et al. Molecular, pathological, radiological, and immune profiling of non-brainstem pediatric high-grade glioma from the HERBY phase II randomized trial. Cancer Cell. 2018;33(5):829-842.e5.

14. Zhang L, Han X, Shi Y. Association of MUC16 mutation with response to immune checkpoint inhibitors in solid tumors. JAMA Netw Open. 2020;3(8):e2013201.

15. Andreiuolo F, Lisner T, Zlocha J, Kramm C, Koch A, Bison B, Gareton A, Zanello M, Waha A, Varlet P, et al. H3F3A-G34R mutant high grade neuroepithelial neoplasms with glial and dysplastic ganglion cell components. Acta Neuropathol Commun. 2019;7(1):78.

16. Sasaki S, Tomomasa R, Nobusawa S, Hirato J, Uchiyama T, Boku E, Miyasaka T, Hirose T, Ohbayashi C. Anaplastic pleomorphic xanthoastrocytoma associated with an H3G34 mutation: a case report with review of literature. Brain Tumor Pathol. 2019;36(4):169-73.

17. Yoshimoto K, Hatae R, Sangatsuda Y, Suzuki SO, Hata N, Akagi Y, Kuga D, Hideki M, Yamashita K, Togao O, et al. Prevalence and clinicopathological features of H3.3 G34-mutant high-grade gliomas: a retrospective study of 411 consecutive glioma cases in a single institution. Brain Tumor Pathol. 2017;34(3):103-12.

18. Wang L, Shao L, Li H, Yao K, Duan Z, Zhi C, Song S, Cheng Y, Wang F, Wang W, et al. Histone H3.3 G34-mutant diffuse gliomas in adults. Am J Surg Pathol. 2021. https://doi.org/10.1097/PAS.0000000000001781.

19. Guerit E, Arts F, Dachy G, Boulouadnine B, Demoulin JB. PDGF receptor mutations in human diseases. Cell Mol Life Sci. 2021;78(8):3867-81.

20. Chojnacki A, Mak G, Weiss S. PDGFRalpha expression distinguishes GFAPexpressing neural stem cells from PDGF-responsive neural precursors in the adult periventricular area. J Neurosci. 2011;31(26):9503-12.

21. Chiang J, Harreld JH, Tanaka R, Li X, Wen J, Zhang C, Boue DR, Rauch TM, Boyd JT, Chen J, et al. Septal dysembryoplastic neuroepithelial tumor: a comprehensive clinical, imaging, histopathologic, and molecular analysis. Neuro Oncol. 2019;21(6):800-8.

22. Paugh BS, Zhu X, Qu C, Endersby R, Diaz AK, Zhang J, Bax DA, Carvalho $D$, Reis RM, Onar-Thomas A, et al. Novel oncogenic PDGFRA mutations in pediatric high-grade gliomas. Cancer Res. 2013;73(20):6219-29.

23. Ozawa T, Brennan CW, Wang L, Squatrito M, Sasayama T, Nakada M, Huse JT, Pedraza A, Utsuki S, Yasui Y, et al. PDGFRA gene rearrangements are frequent genetic events in PDGFRA-amplified glioblastomas. Genes Dev. 2010;24(19):2205-18.

24. Velghe Al, Van Cauwenberghe S, Polyansky AA, Chand D, MontanoAlmendras CP, Charni S, Hallberg B, Essaghir A, Demoulin JB. PDGFRA alterations in cancer: characterization of a gain-of-function V536E transmembrane mutant as well as loss-of-function and passenger mutations. Oncogene. 2014;33(20):2568-76. 
25. Roberts PJ, Der CJ. Targeting the Raf-MEK-ERK mitogen-activated protein kinase cascade for the treatment of cancer. Oncogene. 2007;26(22):3291-310.

26. Lemmon MA, Schlessinger J. Cell signaling by receptor tyrosine kinases. Cell. 2010;141(7):1117-34.

27. Verstraete K, Savvides SN. Extracellular assembly and activation principles of oncogenic class III receptor tyrosine kinases. Nat Rev Cancer. 2012;12(11):753-66.

28. Evans EK, Gardino AK, Kim JL, Hodous BL, Shutes A, Davis A, Zhu XJ, Schmidt-Kittler O, Wilson D, Wilson K, et al. A precision therapy against cancers driven by KIT/PDGFRA mutations. Sci TransI Med. 2017. https:// doi.org/10.1126/scitransImed.aao1690.

29. Heinrich MC, Griffith D, McKinley A, Patterson J, Presnell A, Ramachandran A, Debiec-Rychter M. Crenolanib inhibits the drug-resistant PDGFRA D842V mutation associated with imatinib-resistant gastrointestinal stromal tumors. Clin Cancer Res. 2012;18(16):4375-84.

30. Demetri GD, von Mehren M, Blanke CD, Van den Abbeele AD, Eisenberg B, Roberts PJ, Heinrich MC, Tuveson DA, Singer S, Janicek M, et al. Efficacy and safety of imatinib mesylate in advanced gastrointestinal stromal tumors. N Engl J Med. 2002;347(7):472-80.

31. Loizos N, Xu Y, Huber J, Liu M, Lu D, Finnerty B, Rolser R, Malikzay A, Persaud A, Corcoran E, et al. Targeting the platelet-derived growth factor receptor alpha with a neutralizing human monoclonal antibody inhibits the growth of tumor xenografts: implications as a potential therapeutic target. Mol Cancer Ther. 2005;4(3):369-79.

32. Li H, Shan C, Wu S, Cheng B, Fan C, Cai L, Chen Y, Shi Y, Liu K, Shao Y, et al. Genomic profiling identified novel prognostic biomarkers in Chinese midline glioma patients. Front Oncol. 2020;10:607429.

33. Pathania M, De Jay N, Maestro N, Harutyunyan AS, Nitarska J, Pahlavan P, Henderson S, Mikael LG, Richard-Londt A, Zhang Y, et al. H3.3(K27M) cooperates with Trp53 loss and PDGFRA gain in mouse embryonic neural progenitor cells to induce invasive high-grade gliomas. Cancer Cell. 2017;32(5):684-700.e9.

34. Yang RR, Shi ZF, Zhang ZY, Chan AK, Aibaidula A, Wang WW, Kwan J, Poon WS, Chen H, Li WC, et al. IDH mutant lower grade (WHO grades II/ III) astrocytomas can be stratified for risk by CDKN2A, CDK4 and PDGFRA copy number alterations. Brain Pathol. 2020;30(3):541-53.

35. Dufour C, Perbet R, Leblond P, Vasseur R, Stechly L, Pierache A, Reyns N, Touzet G, Le Rhun E, Vinchon M, et al. Identification of prognostic markers in diffuse midline gliomas H3K27M-mutant. Brain Pathol. 2020;30(1):179-90.

36. Imai $Y$, Yamagishi $H$, Fukuda $K$, Ono $Y$, Inoue $T$, Ueda Y. Differential mucin phenotypes and their significance in a variation of colorectal carcinoma. World J Gastroenterol. 2013;19(25):3957-68.

37. Boltin D, Perets TT, Vilkin A, Niv Y. Mucin function in inflammatory bowel disease: an update. J Clin Gastroenterol. 2013;47(2):106-11.

38. Jiang Z, Wang H, Li L, Hou Z, Liu W, Zhou T, Li Y, Chen S. Analysis of TGCA data reveals genetic and epigenetic changes and biological function of MUC family genes in colorectal cancer. Future Oncol. 2019;15(35):4031-43.

39. Ringel J, Lohr M. The MUC gene family: their role in diagnosis and early detection of pancreatic cancer. Mol Cancer. 2003;2:9.

40. Wierzbicki K, Ravi K, Franson A, Bruzek A, Cantor E, Harris M, Homan MJ, Marini BL, Kawakibi AR, Ravindran R, et al. Targeting and therapeutic monitoring of H3K27M-mutant glioma. Curr Oncol Rep. 2020;22(2):19.

41. Tinkle CL, Broniscer A, Chiang J, Campagne O, Huang J, Orr BA, Li X, Patay Z, Zhang J, Baker SJ, et al. Phase I study using crenolanib to target PDGFR kinase in children and young adults with newly diagnosed DIPG or recurrent high-grade glioma, including DIPG. Neurooncol Adv. 2021;3(1):vdab179.

\section{Publisher's Note}

Springer Nature remains neutral with regard to jurisdictional claims in published maps and institutional affiliations. 